\title{
Motivos para ingresar a las carreras de Pedagogía de los estudiantes de primer año de la Universidad de Concepción*
}

\author{
Motives for entering to the careers of Pedagogy in students of first year at Universidad \\ de Concepción
}

\author{
Motivos para ingressar às carreiras de Pedagogia dos estudantes de primeiro ano da \\ Universidad de Concepción
}

\author{
Cecilia Avendaño Bravo, ${ }^{\text {a }}$ Rodrigo González Urrutia ${ }^{\text {a }}$ \\ aDepartamento de Psicología, Universidad de Concepción. Telf.: 2204323. \\ Correo electrónico: rodrigogonzalez@udec.cl
}

\begin{abstract}
RESUMEN
Esta investigación tuvo como objetivo describir las motivaciones de los estudiantes de primer año de diferentes especialidades de Pedagogía para realizar estudios superiores en estas carreras. La muestra corresponde a estudiantes voluntarios de ambos sexos de 11 carreras de Pedagogía de la Universidad de Concepción. Los datos fueron recogidos mediante un cuestionario. Los resultados muestran que para la mayoría de los estudiantes de primer año la opción de estudiar Pedagogía fue su primera preferencia (65\%). Las tres carreras con más alto porcentaje de estudiantes que las eligieron en primera opción son Pedagogía en Educación Musical, Pedagogía en Español y las Pedagogías en Educación Física y Educación Diferencial. El comportamiento específico de los estudiantes de Pedagogía para postular en primera, segunda, tercera o cuarta opción parece variar según la carrera. Por último, la mayoría de los estudiantes presentan motivos intrínsecos para ingresar a carreras de Pedagogía.
\end{abstract}

Palabras clave: pedagogía, motivación, elección de carrera.

\begin{abstract}
This investigation had as main aim to describe the motivations of first year students of different specialties of Pedagogy to pursue studies in these careers. The sample corresponds to voluntary students of both sexes of 11 careers of Pedagogy at Concepción University. The information was gathered by means of a questionnaire. The results demonstrated that the majority of the students of first year decided to study Pedagogy as their first preference $(65 \%)$. Three careers with higher percentage of students who chose them in the first option are A Pedagogy in Musical Education, Pedagogy in Spanish and the Pedagogies in Physical Education and Differential Education. The specific behavior of the students of Pedagogy to postulate in the first, second, third or fourth option seems to change according to the career. Finally, the majority of the students present intrinsic motives for entering to careers of Pedagogy.
\end{abstract}

Key words: pedagogy, motivation, choice of career.

\section{RESUMO}

Este estudo teve como objetivo descrever as motivações dos alunos do primeiro ano de diferentes áreas da Educação, para realizar estudos com essas raças. A amostra corresponde a estudantes voluntários de ambos os sexos, de 11 raças de Educação da Universidade de Concepción. Os dados foram coletados através de questionário. Os resultados mostram que para a maioria dos estudantes do primeiro ano da faculdade de pedagogia estudar era sua primeira escolha (65\%). As três corridas com o maior percentual de alunos que escolheram a primeira opção é Pedagogia em Educação Musical, Educação em Espanhol e Educação Física Pedagogia e educação especial. O comportamento específico dos alunos da Educação de correr para a primeira opção, segundo, terceiro ou quarto parece variar de acordo com a raça. Finalmente, a maioria dos estudantes têm razões intrínsecas para entrar carreira docente.

Palavras chave: educação, motivação, escolha da carreira. 


\section{INTRODUCCIÓN}

El futuro y el desarrollo de un país dependen directamente del nivel de educación de su gente. A la escuela como institución docente corresponde el papel formal de la educación y es la instancia donde se ejecutan todas las acciones para formar egresados con determinadas cualidades que les permitirán enfrentarse a la vida, ya sea para continuar estudios superiores o entrar al mundo laboral (Rodríguez-González, Peteiro \& Rodríguez-Wong, 2007).

Lo anterior permite entender el rol principal que tienen los docentes en la formación de futuras generaciones. Ellos median y acompañan el proceso por el cual los educandos desarrollan sus conocimientos, sus capacidades, sus destrezas, actitudes y valores, debiendo hacerlo en un marco que valora a los demás y respeta los derechos individuales y sociales (Comisión Nacional de Acreditación, 2007). La evidencia aportada por investigaciones recientes sugiere que los buenos maestros marcan una clara diferencia en los aprendizajes que logran sus alumnos, en sus rendimientos y, en definitiva, en el éxito escolar que estos alumnos puedan tener (Latorre, 2005).

Sin embargo, las condiciones de trabajo de los docentes chilenos son altamente demandantes y exigentes. Sus condiciones laborales presentan falta de recursos para realizar el trabajo, alta exposición a relaciones interpersonales con los diferentes agentes de la comunidad educativa, y exigencias de alumnos, apoderados, directivos, instituciones de evaluación externa, entre otras, que conllevan tensiones que pueden derivar en estrés laboral y consecuencias dañinas sobre su salud y desempeño laboral (Parra, 2005). En general, cumplen además un excesivo número de horas de clases en relación con sus horas de contrato, lo cual limita sus posibilidades de dedicar el tiempo necesario a la preparación de clases, el estudio, la reflexión y el trabajo en equipo, y la atención de padres y alumnos, entre otras tareas esenciales de la función pedagógica (Comisión sobre Formación Inicial Docente, 2005). Por otra parte, aunque sus remuneraciones han ido mejorando a través de los años, las posibilidades de aumentarlas a lo largo de la carrera laboral son muy limitadas (Comisión sobre Formación Inicial Docente, 2005).

En este escenario complejo, tener los profesores más motivados con la Pedagogía para llevar a cabo la misión educadora, es fundamental, porque ello influirá directamente en los aprendizajes de sus alumnos.

Diversas investigaciones destacan las siguientes motivaciones de los profesores por la profesión docente: querer trabajar con niños y/o jóvenes, gusto por enseñar, gusto por la especialidad, gusto por la educación (Bertomeu et al., 2007). En Chile, un estudio con docentes realizado por el Ministerio de Educación en conjunto con la Universidad de Chile (2005) identifica los siguientes motivos para haber elegido su carrera: les gusta enseñar a niños y jóvenes (57\%), la educación tiene la función más importante dentro de la sociedad (56\%) y, por último, le gusta la asignatura que hoy enseña (53\%), aunque sólo un 34\% tenía interés por la carrera en Cuarto Medio y menos del 50\% de los docentes activos tenía como primera prioridad estudiar Pedagogía. Por otra parte, en un estudio de Mizala y Romaguera (2001) se observó que un 48\% de los estudiantes de Pedagogía manifestaban una fuerte vocación por su carrera -medidos a través del interés manifestado de que sus hijos estudien la misma carrera-, frente a un $35.2 \%$ de estudiantes de otras carreras enfrentados a la misma situación. 
En general, la mayoría de las investigaciones que indagan sobre motivaciones hacia la Pedagogía ocupan muestras de profesionales docentes y son escasos aquellos específicamente orientados a conocer las motivaciones de los estudiantes de Pedagogía para ingresar a estudiar una carrera en esta área.

Según Pérez y Blasco (2001), en el ingreso a una carrera determinada subyace una opción o inclinación motivada por diversas razones hacia un área de trabajo ocupación o profesión, que daría cuenta del porqué una persona desea acercarse y profundizar aún más en ésta, llegando en la mayoría de los casos a ser la profesión de vida. La elección de la carrera profesional es una acción que involucra la toma de una decisión trascendental y, en la mayoría de los casos, irreversible. No obstante, diversas investigaciones indican que los jóvenes no sólo eligen una carrera por motivos propios, sino que su decisión está mediada por múltiples factores tales como la personalidad, los elementos de su entorno (influencia familiar, modelos de referencia, tendencias sociales, etc.), el deseo de satisfacer necesidades, entre otros (Pérez \& Blasco, 2001). Entre las diversas teorías sobre la motivación, aquellas que hablan de la motivación extrínseca se refieren precisamente a la motivación relacionada con el tipo de fuerzas que mueven y dirigen las acciones de las personas en función de las recompensas externas que pueda recibir (Deci \& Ryan, 2000).

En el caso de los docentes, se ha señalado que quienes eligen la carrera docente motivados intrínsecamente, tendrán mayor compromiso y eficiencia que quienes lo hicieron por motivos extrínsecos como razones económicas, tradición familiar u otros (García, 2007). Este planteamiento es defendido por la Teoría de la Autodeterminación, que plantea que los individuos cuya elección ha sido determinada por motivos extrínsecos o está regulada por recompensas externas, están menos comprometidos con su desempeño y tienen menos confianza en lo que hacen (Deci \& Ryan, 2000).

Sin embargo, Moreno (2001) señala que algunos estudios sobre motivación de ingreso a Pedagogía indican que muchos estudiantes no tienen motivación intrínseca por la carrera, pero muestran intereses cognoscitivos y profesionales hacia las respectivas disciplinas. Añade que, casi siempre, quienes optan por la pedagogía son estudiantes con menos posibilidades académicas para otras carreras de mayor reconocimiento y valoración social, y muchas veces están gobernados por alguna razón personal o por presión de sus familias a estudiar una carrera universitaria.

Otros estudios señalan que la elección profesional varía según el género (Sáinz, López-Sáez \& Lisbona, 2004), así, existirían carreras típicamente masculinas y femeninas. Sáinz et al. concluyeron que en el caso de las mujeres la motivación para elegir una carrera hace referencia a la vocación y a factores intrínsecos de interés por el contenido y ayuda a los demás, destacando entre éstas las carreras sociales y de humanidades; en el caso de los hombres, las carreras serían elegidas por motivos más instrumentales tales como el salario, las salidas profesionales y la proyección profesional, destacándose entre las carreras elegidas las de orden científico y técnico. En nuestro país, la mayoría de los estudiantes motivados hacia la Pedagogía son mujeres (Bravo, Peirano \& Falck, 2006).

En la perspectiva de contribuir a ampliar el conocimiento en esta materia en nuestro país, la investigación que aquí se reporta estuvo dirigida principalmente a describir los motivos de estudiantes de primer año de la Universidad de Concepción para ingresar a estudiar Pedagogía, según sexo, especialidad elegida y opción por la carrera. 
Los resultados de esta investigación aportan información sobre el perfil motivacional de los alumnos que ingresan a estudiar diferentes especialidades pedagógicas, el cual puede ser de utilidad para orientar el currículo inicial de la formación de profesores, especialmente en las asignaturas que permiten fortalecer la dimensión vocacional entre los futuros enseñantes.

\section{METODOLOGÍA}

Para lograr el objetivo propuesto se realizó un estudio con diseño transversal de tipo descriptivo. La muestra está constituida por 279 estudiantes de 11 carreras de Pedagogía de la Facultad de Educación de la Universidad de Concepción, lo que representa un 64\% del total de estudiantes matriculados en el primer año de estas carreras en el 2009. Un $67 \%$ son mujeres y el $33 \%$ restante hombres, en un rango de 18 a 31 años de edad (media de 19 años). El detalle por carrera puede observarse en la siguiente tabla (Tabla 1).

\begin{tabular}{|c|c|c|c|c|}
\hline \multirow{2}{*}{} & \multicolumn{2}{|c|}{ Matrícula } & \multicolumn{2}{c|}{ Participantes } \\
\hline $\begin{array}{c}\text { Carrera y/o } \\
\text { Especialidades }\end{array}$ & fr & \% & fr & \% \\
\hline Educación Diferencial & 48 & 11 & 37 & 77 \\
\hline Filosofía & 14 & 3 & 11 & 79 \\
\hline Español & 37 & 9 & 37 & 100 \\
\hline $\begin{array}{c}\text { Matemáticas y } \\
\text { Computación }\end{array}$ & 32 & 7 & 12 & 38 \\
\hline Educación General Básica & 99 & 23 & 32 & 32 \\
\hline Educación Física & 69 & 16 & 47 & 68 \\
\hline Educación Parvularia & 39 & 9 & 21 & 54 \\
\hline Educación Musical & 23 & 5 & 18 & 78 \\
\hline $\begin{array}{c}\text { Ciencias Naturales y } \\
\text { Biología }\end{array}$ & 24 & 6 & 18 & 75 \\
\hline Ciencias Naturales y Física & 22 & 5 & 20 & 94 \\
\hline Ciencias Naturales y \\
Química
\end{tabular}

Tabla 1. Matrícula de primer año de Pedagogía en la UdeC y participantes en el estudio, en frecuencias y porcentajes 


\section{INSTRUMENTOS}

Para recoger la información se utilizó el cuestionario "Motivación hacia estudios del Magisterio", desarrollado en el marco de una investigación descriptiva sobre motivaciones de ingreso a carreras de educación realizada por investigadores de las Universidades Jaume I de Castellón y la Universitat de Valencia (Bertomeu et al., 2007). Este instrumento fue adaptado en aquellas expresiones o palabras típicamente españolas de poco uso en nuestro país. Consta de 26 ítems que reflejan las distintas motivaciones para entrar al magisterio, entre las cuales el/la participante debe elegir tres y, luego, jerarquizarlas según la importancia que les otorgue. Además, a cada participante se le solicitó que informará la opción en que postuló a Pedagogía (primera, segunda, tercera o cuarta opción); sexo; edad y especialidad que estudia.

\section{PROCEDIMIENTO}

Primero, se tomó contacto con docentes que imparten asignaturas troncales en las distintas especialidades/carreras de Pedagogía con el propósito de obtener su autorización para aplicar el instrumento a sus estudiantes en sus aulas. La aplicación fue colectiva en diversas aulas de la Universidad de Concepción. Para esta aplicación se obtuvo el consentimiento y la participación voluntaria de los estudiantes.

\section{ANÁLISIS DE LOS DATOS}

Para el análisis de datos fue utilizada estadística descriptiva, siendo calculadas medidas de tendencia central y de dispersión. Los datos fueron procesados con el software estadístico SPSS versión 15.0.

\section{RESULTADOS}

A continuación se describen los principales resultados obtenidos en el análisis de los datos. En primer lugar se presentan los resultados relacionados con la opción por la carrera de Pedagogía a que ingresaron y, luego, los resultados vinculados con los motivos que los estudiantes tuvieron para ingresar a estudiar Pedagogía.

\section{1. OPCIÓN POR PEDAGOGÍA}

El 65\% de los estudiantes postuló a una carrera de Pedagogía en primera opción; $22 \%$ postuló en segundo lugar a esa carrera; un $10 \%$ lo hizo en tercer lugar, y para un $3 \%$ fue su cuarta opción. Destacan la postulación a Pedagogía en Educación Musical en la cual el $100 \%$ de los estudiantes postularon en primera o segunda opción (83\% y $27 \%$, respectivamente), y Pedagogía en Español con un $94 \%$ en primera y segunda opción. Pedagogía en Filosofía representa un caso particular debido a que los estudiantes 
matriculados en primer año postularon en casi igual proporción en primer, segundo y tercer lugar de preferencia.

Más detalles pueden apreciarse en la Tabla 2.

\begin{tabular}{|c|c|c|c|c|c|c|c|c|}
\hline \multicolumn{7}{|c|}{ Estudiantes en cada opción } \\
\hline & Primera & \multicolumn{2}{|c|}{ Segunda } & \multicolumn{2}{c|}{ Tercera } & \multicolumn{2}{c|}{ Cuarta o + } \\
\hline Carrera & $\mathbf{f r}$ & $\mathbf{\%}$ & $\mathbf{f r}$ & $\mathbf{\%}$ & $\mathbf{f r}$ & $\mathbf{\%}$ & $\mathbf{f r}$ & $\mathbf{\%}$ \\
\hline Educación Diferencial & 26 & 70 & 9 & 24 & 2 & 6 & 0 & 0 \\
\hline Filosofía & 4 & 36 & 4 & 36 & 3 & 28 & 0 & 0 \\
\hline Español & 29 & 78 & 6 & 16 & 1 & 3 & 1 & 3 \\
\hline $\begin{array}{c}\text { Matemáticas y } \\
\text { Computación }\end{array}$ & 8 & 67 & 3 & 25 & 1 & 8 & 0 & 0 \\
\hline Pedagogía General Básica & 17 & 53 & 8 & 25 & 6 & 19 & 1 & 3 \\
\hline Educación Física & 33 & 70 & 9 & 19 & 4 & 9 & 1 & 2 \\
\hline Educación Parvularia & 14 & 68 & 5 & 24 & 1 & 4 & 1 & 4 \\
\hline Educación Musical & 15 & 83 & 3 & 17 & 0 & 0 & 0 & 0 \\
\hline $\begin{array}{c}\text { Ciencias Naturales y } \\
\text { Biología }\end{array}$ & 10 & 56 & 4 & 22 & 3 & 17 & 1 & 6 \\
\hline Ciencias Naturales y Física & 13 & 65 & 5 & 25 & 1 & 5 & 1 & 5 \\
\hline $\begin{array}{c}\text { Ciencias Naturales y } \\
\text { Química }\end{array}$ & 12 & 46 & 5 & 19 & 7 & 27 & 2 & 8 \\
\hline Total & $\mathbf{1 8 1}$ & $\mathbf{6 5}$ & $\mathbf{6 1}$ & $\mathbf{2 2}$ & $\mathbf{2 9}$ & $\mathbf{1 0}$ & $\mathbf{8}$ & $\mathbf{3}$ \\
\hline
\end{tabular}

Tabla 2. Distribución de los estudiantes en las carreras de Pedagogía según la opción en que postuló al ingresar, en frecuencias y porcentajes

El porcentaje más alto de postulantes a Pedagogía en primera opción es de hombres, con un $70 \%$. En segunda y cuarta opción las diferencias por sexo en los porcentajes son mínimas, pero, en tercera opción, la proporción de mujeres es más del doble que la de hombres. Por edad, los datos señalan que el $76 \%$ de los estudiantes que postularon en todas las opciones se ubican en el rango de 18 a 20 años. En la muestra, a medida que aumenta la edad es menor la postulación a Pedagogía $(\mathrm{F}=1,139$, sig. ,328). La elección de la carrera de Pedagogía en segunda opción concentra la mayor proporción de estudiantes sobre los 20 años de edad (30\%), seguida por un 24\% en primera opción, un 4\% en tercera opción y un $1 \%$ en cuarta o más. Estos resultados llevan a hipotetizar que en su mayoría los estudiantes de primer año de Pedagogía están cercanos al momento de egreso de la Enseñanza Media.

\section{2. MOTIVOS PARA ESTUDIAR PEDAGOGÍA}

En general, los cinco motivos más elegidos para ingresar a la carrera, sin importar la opción declarada, son los siguientes: 
1. "Me gustaba mi especialidad", con un $50 \%$ del total de respuestas de los estudiantes y elegida en primera opción por un $25 \%$.

2. "Me gustaba la enseñanza", elegida por un $39 \%$ del total de respuestas de los estudiantes y en primera opción por un $18 \%$.

3. "Pensaba que sería gratificante educar a jóvenes o niños pequeños", con $38 \%$ del total de respuestas de los estudiantes y un $10 \%$ como principal motivación.

4. "Deseaba ayudar a los demás", con un $28 \%$ del total de respuestas de los estudiantes y un $9 \%$ en primera opción.

5. "Me gustaban los niños y/o trabajar con ellos", con un $8,2 \%$ del total de respuestas de los estudiantes y un $8 \%$ como el primer motivo para ingresar a Pedagogía.

Por el contrario, los cinco motivos menos elegidos $-\mathrm{o}$ no elegidos- para ingresar a la carrera son los siguientes:

1. "Pensaba que era una carrera corta", que no fue elegida por ningún estudiante.

2. "Por tradición familiar", que no fue elegida por ningún estudiante.

3. "Deseaba ocupar el tiempo", que no fue elegida por ningún estudiante en primera opción, y sólo por 1 como segunda opción y por 1 como tercera opción.

4. "Porque alguien muy relacionado conmigo eligió esta carrera también", elegida por 1 estudiante en tercera opción.

5. "Por razones económicas", elegida por 2 estudiantes en primera opción y 2 en segundo opción.

En primera opción, los motivos más frecuentes para estudiar una carrera en Pedagogía aparecen más concentrados entre los hombres que entre las mujeres. Los motivos más frecuentemente señalados por los hombres son: Me gustaba mi especialidad (26\%); Me gustaba la enseñanza (21\%). Entre las mujeres son los siguientes: Me gustaba mi especialidad (25\%); Me gustaba la enseñanza (17\%); Pensaba que sería gratificante educar a jóvenes o niños pequeños (13\%) y Me gustaban los niños y/o trabajar con ellos (11\%).

\subsubsection{Motivos por carrera}

Los motivos que los estudiantes tuvieron para elegir su carrera en primera opción no son muy variados, por el contrario, los datos dan cuenta de homogeneidad en las respuestas. Además, por especialidad se observa una cierta coherencia con el primer motivo declarado y la naturaleza de los estudios elegidos. De este modo, casi siempre la frecuencia más alta la obtiene la alternativa "Me gustaba mi especialidad". Es notoria Educación Musical donde la mayoría de las respuestas de los estudiantes está concentrada en una sola alternativa, esto es, "Me gustaba mi especialidad".

La alternativa "No pude acceder a otra carrera" aparece mencionada con una frecuencia relativamente importante sólo entre los estudiantes de las siguientes tres carreras: Pedagogía en Educación Física y dos del área de las ciencias naturales, Pedagogía en Ciencias Naturales y Biología y Pedagogía en Ciencias Naturales y Química, sin embargo, en ninguna es la alternativa que concentra la frecuencia más alta.

Los motivos más frecuentemente mencionados para estudiar la carrera en que están formándose actualmente son los siguientes: 


\begin{tabular}{|c|c|}
\hline Carrera & Motivos principales \\
\hline $\begin{array}{l}\text { Educación } \\
\text { Diferencial }\end{array}$ & $\begin{array}{c}\text {-Me gustaban los niños y/o trabajar con ellos }(28 \%) \\
\text {-Pensaba que sería gratificante educar a jóvenes o niños } \\
\text { pequeños ( } 22 \%) \\
\text {-Me gustaba la enseñanza, Me gustaba mi especialidad y } \\
\text { Deseaba ayudar a los demás (14\% respectivamente) }\end{array}$ \\
\hline Filosofía & $\begin{array}{c}\text {-Me gustaba mi especialidad (27\%) } \\
\text {-Deseaba aprender y Deseaba ayudar a los demás (18\% } \\
\text { respectivamente) }\end{array}$ \\
\hline Español & $\begin{array}{c}\text {-Me gustaba mi especialidad (24\%) } \\
\text {-Me gustaba la enseñanza (19\%) } \\
\text {-Pensaba que sería gratificante educar a jóvenes o niños } \\
\text { pequeños y Consideraba mi especialidad un aspecto } \\
\text { fundamental para la formación (11\% respectivamente) }\end{array}$ \\
\hline $\begin{array}{l}\text { Matemáticas y } \\
\text { Computación }\end{array}$ & $\begin{array}{l}\text {-Me gustaba mi especialidad (33\%) } \\
\text {-Me gustaba la enseñanza y Pensaba que sería gratificante } \\
\text { educar a jóvenes o niños pequeños (17\% respectivamente) }\end{array}$ \\
\hline $\begin{array}{l}\text { Pedagogía General } \\
\text { Básica }\end{array}$ & $\begin{array}{c}\text {-Me gustaban los niños y/o trabajar con ellos }(28 \%) \\
\text {-Pensaba que sería gratificante educar a jóvenes o niños } \\
\text { pequeños }(19 \%) \\
\text {-Me gustaba todo lo relacionado con el ámbito educativo }(16 \%)\end{array}$ \\
\hline Educación Física & $\begin{array}{l}\text {-Me gustaba mi especialidad }(34 \%) \\
\text {-Me gustaba la enseñanza }(22 \%) \\
\text {-No pude acceder a otra carrera }(11 \%)\end{array}$ \\
\hline $\begin{array}{l}\text { Educación } \\
\text { Parvularia }\end{array}$ & $\begin{array}{c}\text {-Me gustaba mi especialidad y Deseaba ayudar a los demás } \\
\text { (24\% respectivamente) } \\
\text {-Pensaba que sería gratificante educar a jóvenes o niños } \\
\text { pequeños }(14 \%)\end{array}$ \\
\hline Educación Musical & -Me gustaba mi especialidad (56\%) \\
\hline $\begin{array}{c}\text { Ciencias Naturales } \\
\text { y Biología }\end{array}$ & $\begin{array}{c}\text {-Me gustaba la enseñanza (33\%) } \\
\text {-Me gustaba mi especialidad (22\%) } \\
\text {-Pensaba que sería gratificante educar a jóvenes o } \\
\text { niños pequeños y No pude acceder a otra carrera (17\% } \\
\text { respectivamente) } \\
\end{array}$ \\
\hline $\begin{array}{c}\text { Ciencias Naturales } \\
\text { y Física } \\
\end{array}$ & $\begin{array}{l}\text {-Me gustaba la enseñanza (30\%) } \\
\text {-Me gustaba mi especialidad (25\%) }\end{array}$ \\
\hline $\begin{array}{c}\text { Ciencias Naturales } \\
\text { y Química }\end{array}$ & $\begin{array}{l}\text {-Me gustaba mi especialidad (31\%) } \\
\text {-Me gustaba la enseñanza }(27 \%) \\
\text {-No pude acceder a otra carrera }(23 \%)\end{array}$ \\
\hline
\end{tabular}

Cuadro 1. Principales motivos señalados por carrera 


\subsubsection{Motivos intrínsecos o extrínsecos para elegir una carrera}

Cuando los motivos son analizados, según si corresponden a motivos intrínsecos o extrínsecos, los datos permiten afirmar que en un $60 \%$ las respuestas de los estudiantes están asociadas con aspectos positivos para ingresar a estudios en Pedagogía que implican una motivación intrínseca (por ejemplo, "Pensaba que sería gratificante educar a jóvenes o niños pequeños"). Si a los motivos anteriores se agrega el subgrupo referido a motivos vinculados con características personales ajenas a la Pedagogía, pero que constituyen motivaciones intrínsecas (por ejemplo, "Deseaba aprender"), el porcentaje se eleva a un $65 \%$.

Entre los motivos extrínsecos, el primer lugar (12\%) corresponde al subgrupo de motivos ajenos a la Pedagogía vinculados con la posibilidad de realizar estudios universitarios, que constituyen en general motivaciones extrínsecas para ingresar a una carrera de Pedagogía (por ejemplo, "Deseaba obtener un título universitario sin importar cuál"). En segundo lugar (8\%) se ubican los motivos vinculados con una percepción positiva de tipo instrumental sobre los estudios de Pedagogía o con las condiciones de trabajo docente (por ejemplo, "Creía que era una profesión con buenas oportunidades de trabajo"). En tercer lugar (4,5\%) aparecen los motivos extrínsecos vinculados con la influencia de otros significativos en relación con los estudios en Pedagogía (por ejemplo, "Por imposición familiar").

\section{DISCUSIÓN Y CONCLUSIONES}

Esta investigación tuvo como propósito responder la siguiente interrogante: ¿Cuáles son los motivos que tienen los estudiantes de Pedagogía para ingresar a la carrera? Específicamente, se intentó identificar la prioridad otorgada por estudiantes de primer año de 11 carreras de Pedagogía de la Universidad de Concepción a su opción por estas carreras y los principales motivos que tuvieron para seguir estos estudios. Asimismo, la investigación estuvo centrada en la comparación de sus motivaciones de ingreso según sexo, especialidad elegida y prioridad en la postulación.

La muestra, constituida por 279 estudiantes matriculados en primer año en carreras de Pedagogía en la Universidad de Concepción, tiene en promedio 19 años de edad, no obstante, es destacable que un $23 \%$ tiene entre 21 y 31 años de edad. Esto último permite hipotetizar que, al menos, casi un cuarto de los estudiantes de primer año de Pedagogía no ingresa en forma directa a su actual carrera al salir de cuarto medio. Además, la mayoría de la muestra son mujeres (67\%) y, por lo mismo, el ingreso a casi todas las Pedagogías de la Universidad de Concepción aparece feminizado, ya sea porque ingresan en forma exclusiva mujeres (por ejemplo, en Pedagogía en Educación Diferencial y Educación Parvularia) o principalmente mujeres (por ejemplo, en Pedagogía en Filosofía, Pedagogía en Español y Pedagogía General Básica), observándose que solamente en Educación Musical el ingreso de hombres es mayor que el de mujeres. Esta feminización concuerda con los datos oficiales sobre ingreso a Pedagogía en el nivel nacional (CRUNCH, 2010). Según Barberá (1998), desde una perspectiva de género, la probabilidad de que las mujeres sean docentes es alta porque estaría dentro de las profesiones más feminizadas, dado que es considerada una actividad vinculada con lo social y con la intención de ayudar 
a los demás o entregar asistencia al otro, apropiada a las características tradicionales atribuidas a las mujeres.

En términos generales se observó que para la gran mayoría de los estudiantes de primer año la opción de estudiar Pedagogía fue su primera preferencia (65\%). El 35\% restante se distribuye entre quienes lo hicieron en segunda opción (22\%), tercera opción $(10 \%)$ o cuarta opción (3\%). También se observó que, así como en todas las carreras hay estudiantes de primer año de Pedagogía que postularon a su carrera en primera opción, también en todas hay quienes lo hicieron en segunda y tercera opción, mientras que sólo en cuatro carreras no hubo preferencia de ningún participante en cuarta opción (Educación Diferencial, Filosofía, Matemáticas y Computación y Educación Musical). Las tres carreras con más alto porcentaje de estudiantes que las eligieron en primera opción son Pedagogía en Educación Musical (83\%), Pedagogía en Español (78\%) y, en tercer lugar, las Pedagogías en Educación Física y Educación Diferencial (70\%). Por el contrario, los porcentajes más bajos en primera opción se presentan en las carreras de Pedagogía en Filosofía (36\%), Pedagogía en Ciencias Naturales y Química (46\%), Pedagogía General Básica (53\%) y Pedagogía en Ciencias Naturales y Biología (56\%). En segunda opción, Pedagogía en Filosofía es la carrera que presenta la mayor proporción de estudiantes (36\%). En tercera opción los porcentajes más altos son para las carreras de Pedagogías en Filosofía y Ciencias Naturales y Química (27\%), seguidas de Pedagogía General Básica (19\%) y Pedagogía en Ciencias Naturales y Biología (17\%). Finalmente, en cuarta opción el mayor porcentaje está en las pedagogías que forman el área científica: Ciencias Naturales y Química con un 8\%, seguida por Ciencias Naturales y Física y Ciencias Naturales y Biología con un 5\%.

Llama la atención que el porcentaje de hombres que elige una carrera de Pedagogía en primera opción es superior al porcentaje de mujeres (70\% y 63\%). Por edad, en la muestra se observa que en primera opción por la carrera de Pedagogía la mayoría está concentrada entre los 18 y 20 años (76\%), mientras que en la segunda opción se concentra la mayor proporción de estudiantes sobre los 20 años de edad de la muestra (30\%). Es posible que ello se deba al puntaje en la PSU y a la disminución de probabilidades de volver a postular a una carrera universitaria.

En síntesis, aunque la mayoría optó por una carrera de Pedagogía en primer lugar, la proporción de estudiantes que ingresa en segunda, tercera o cuarta opción pareciera relativamente alta (35\%). Sin embargo, estos resultados resultan más alentadores que los obtenidos por el estudio con docentes llevado a cabo el año 2005 por el Ministerio de Educación de Chile y la Universidad de Chile, que indicaba que, en términos vocacionales, menos del 50\% de los docentes activos tenía como primera prioridad estudiar Pedagogía, y que los resultados recientes del estudio sobre la valoración de la carrera pedagógica realizado por Adimark y Elige Educar (2010,) que dicen que sólo estudiarían pedagogía un $39 \%$ de los jóvenes entre 18 a 24 años, es decir, que quienes estaban por elegir una futura profesión veían la pedagogía como una carrera de interés.

Además, el comportamiento específico de los estudiantes de Pedagogía para postular en primera, segunda, tercera o cuarta opción parece variar según la carrera de qué se trata. Desde este punto de vista, dos resultados llaman la atención: el caso de Educación Musical y el caso de las carreras pedagógicas de orientación "científica". El primero porque aparece en primera opción para la gran mayoría y porque es la única carrera donde predominan los hombres, lo cual conduce a hipotetizar una relación entre ambas 
variables que sería de interés estudiar a futuro desde la perspectiva de la relación entre género y elección vocacional. En el segundo caso, las carreras de orientación científica, especialmente Ciencias Naturales y Química y Ciencias Naturales y Biología, que están entre las menos elegidas en primera opción, y por tanto aparecen más veces en tercera y cuarta opción, y donde predominan las mujeres, a diferencia de Ciencias Naturales y Física en que los hombres representan el 50\% (y en que el 65\% la elige en primera opción y el $25 \%$ en segunda opción), cabe preguntarse qué hace a las dos primeras carreras menos atractivas para postulantes hombres y más para mujeres interesados en pedagogías con orientación científica.

Por otro lado, la gran mayoría de las respuestas de los estudiantes están asociadas con aspectos positivos para ingresar a estudios en Pedagogía que implican una motivación intrínseca (60\%), especialmente "Me gustaba mi especialidad", "Me gustaba la enseñanza", "Pensaba que sería gratificante educar a jóvenes o niños pequeños", "Deseaba ayudar a los demás" y "Me gustaban los niños y/o trabajar con ellos". A su vez, los motivos menos declarados son todos de carácter extrínseco a la Pedagogía, e incluso dos motivos extrínsecos a la Pedagogía no fueron elegidos por ningún participante, y cuando estos motivos son analizados en categorías, se observa que primero aparece mencionado el subgrupo de motivos ajenos a la Pedagogía vinculados con la posibilidad de realizar estudios universitarios en general, luego, los motivos vinculados con una percepción positiva de tipo instrumental sobre los estudios de Pedagogía o con las condiciones de trabajo docente y, por último, los motivos extrínsecos vinculados con la influencia de otros significativos en relación con los estudios en Pedagogía. En relación con las motivaciones extrínsecas, tal vez la Pedagogía sea percibida por muchos de ellos como una posibilidad de adquirir un estatus que les permita movilidad social respecto de su familia de origen.

Los resultados anteriores hacen posible estimar que en su desempeño laboral futuro como docente la mayoría tendría una disposición favorable a valorar contenidos asociados al trabajo docente, estaría dispuesta a involucrarse afectivamente con la actividad y a tratar de ser eficientes en ésta, encontrando recompensa en su ejercicio (Deci y Ryan, 2000; Barberá, 2002; García, 2007). No obstante, para un porcentaje importante que presenta motivos extrínsecos, debiera esperarse un alto riesgo de fracaso en sus estudios o un mal desempeño docente si terminan la carrera (Cano, 2008), si no encuentran incentivos externos apropiados. Cabe señalar que aunque la mayoría de los estudiantes señala motivos intrínsecos, ya sea en primer, segundo o tercer lugar, algunos de ellos presentan una combinación con predominancia de motivos intrínsecos y presencia de algún motivo extrínseco (casi siempre en segunda o tercera preferencia), asunto que debiera ser analizada en futuros estudios.

Finalmente, los resultados muestran que existe una tendencia a la homogeneidad entre los motivos dados en las respuestas de los estudiantes de primer año en las diferentes carreras y que, en general, evidencian cierta congruencia con la especialidad elegida. No aparecen, por otra parte, resultados muy diferentes por sexo, y sólo se observa una tendencia a que las respuestas aparezcan más concentradas entre los hombres lo cual puede ser un efecto de las características de la muestra.

En conclusión, respecto al objeto de estudio de esta investigación, "las motivaciones de ingreso a la carrera pedagógica", la mayoría de las motivaciones de los estudiantes de Pedagogía de la Universidad de Concepción reflejan haber ingresado a su carrera por motivaciones intrínsecas que obedecen a la propia satisfacción o búsqueda de autorrealización. 
Esta circunstancia hace personas con mejores posibilidades de enfrentar la profesión docente, pese a las conocidas dificultades que se presentan durante su ejercicio. Vale comentar que el impacto del nuevo incentivo Beca Vocación de Profesor, podrá verse en un futuro en las generaciones inmediatamente siguientes a la aquí estudiada.

No obstante, dado que las motivaciones por la Pedagogía van mutando, e incluso algunas podrían desaparecer, al enfrentarse con una práctica docente que no siempre cumple con las expectativas iniciales de los profesores, realizar a futuro este estudio en forma longitudinal podría entregar más información relevante. Se sugiere tomar los datos en primer año, por la cercanía que tienen los estudiantes respecto de la razón de su elección; en tercer año, para conocer si la formación docente tiñe en algún grado positivo o negativo esta decisión inicial y, al final de la carrera, puesto que el estudiante tuvo la posibilidad de realizar su práctica profesional, aprobó su formación académica y tiene una visión más amplia de lo que realmente es ser profesor/a. Por último, sería conveniente obtener datos al finalizar el primer año de ejercicio de la profesión debido a que es entonces donde el docente realiza importantes ajustes entre sus expectativas y motivaciones iniciales, que no siempre son compatibles y que en muchos casos pueden causar desgaste excesivo e, incluso, deserción.

A partir de los hallazgos obtenidos en esta investigación, se considera también necesario profundizar en las motivaciones de ingreso a carreras de educación que tienen los estudiantes, considerando como variables el género, la puntuación en la PSU y la situación socioeconómica de los estudiantes de Pedagogía. Además, antes se fueron proponiendo algunas hipótesis correlacionales y explicativas que pudieran orientar futuros estudios que superen las limitaciones de diseño de éste. Otras investigaciones debieran también incluir las carreras que fueron excluidas por razones ajenas a nuestra voluntad (entre éstas, Pedagogía en Historia y Geografía) y comparar todas las carreras por áreas de la Pedagogía en las variables mencionadas. Asimismo, sería interesante comparar los motivos de estudiantes de Pedagogía de universidades tradicionales y no tradicionales, públicas y privadas.

\section{REFERENCIAS BIBLIOGRÁFICAS}

Adimark \& Elige Educar. (2010). Estudio sobre valoración de la profesión docente en Chile. En línea; disponible en http://www.radiobiobio.cl/2010/11/10/estudio-de-adimark-revela-quepedagogias-tienen-baja-valoracion-entre-jovenes-y-estratos-altos/ (visitado noviembre 2010).

Barberá, E. (2002). Modelos explicativos en Psicología de la motivación. Revista Electrónica de Motivación y Emoción, Vol. 5, n. 10. En línea; disponible en http://reme.uji.es/articulos/ abarbe7630705102/texto.html (visitado el 15 de enero de 2010 desde http://reme.uji.es/articulos/).

Bertomeu, F., Canet, G., Gil, V. \& Jarabo, J. (2007). Las Motivaciones hacia los estudios de Magisterio. Ponencia presentada en las Jornadas de Fomento de la Investigación, Universidad de Jaume I de Castellón y Universidad de Valencia. Extraído el 15 de agosto de 2009 desde http://www.uji.es/bin/publ/edicions/jfi12/32.pdf

Bravo, D., Peirano, C. \& Falck, D. (2006). Encuesta Longitudinal de Docentes 2005. Análisis y principales resultados. Santiago, Chile: Universidad de Chile, Departamento de Economía, Centro de Microdatos. 
Comisión Nacional de Acreditación [CNA] (2007). Criterios de evaluación de carreras de Educación. Santiago, Chile: CNA. Extraído el 25 de octubre desde 2009 de http://www.cnachile.cl/materiales/materiales.html

Cano, M. (2008). Motivación y Elección de Carrera. Revista Mexicana de Orientación Educativa, v. 5, n.13, 6-9. Extraído el 20 de agosto de 2009 desde http://pepsic.bvs-psi.org.br/scielo. php?script=sci_arttext\&pid=S1665-75272008000100003\&lng=pt\&nrm=

Consejo de Rectores de Universidades Chilenas [CRUNCH] (2007- 2010). Página web: www. crunch.cl.

Deci, E. L. \& Ryan, R. M. (2000). The "What" and "Why" of Goal Pursuits: Human Needs and the Self-determination of Behavior. Psychological Inquiry, n.11, 227-268.

García, J. M. (2007). Motivación y actitudes hacia la carrera de profesor de educación primaria en estudiantes normalistas de primer ingreso. Revista Mexicana de Investigación Educativa, vol. 12, n. 35. Extraído el 16 de abril de 2008 desde http://redalyc.uaemex.mx/pdf/140/14003503.pdf

Latorre, M. (2005). Continuidades y rupturas entre formación inicial y ejercicio profesional docente. Revista Iberoamericana de Educación, vol. 2, n.36. Extraído el 11 de mayo de 2008 desde http://www.rieoei.org/deloslectores/1049Latorre.PDF

Ministerio de Educación (2005). Informe Comisión sobre Formación Inicial Docente Serie Bicentenario. Chile: Ministerio de Educación.

Ministerio de Educación (2005). Encuesta Longitudinal de Docentes. Análisis y principales resultados. Chile: Ministerio Educación.

Mizala, A. y Romaguera, P. (2001). Regulación, Incentivos y Remuneraciones de los Profesores en Chile. Chile: Universidad de Chile. Serie Economía $\mathrm{N}^{\circ} 116$.

Moreno, M. (2001). Referentes para la orientación y reorientación de la motivación profesional pedagógica. Ciencia y Sociedad, vol. XXVI. Extraído el 12 de marzo de 2010 desde http:// redalyc.uaemex.mx/src/inicio/ArtPdfRed.jsp?iCve=87011300005

Parra, M. (2005). Estudio de caso en Chile. En M. Robalino y A. Körne (Eds.), Condiciones de trabajo y salud docente (pp. 73-100). Santiago, Chile: UNESCO.

Pérez Boullosa, A. \& Blasco Calvo, P. (2001). Orientación e Inserción Profesional: Fundamentos y Tendencias. Valencia. Nau Llibres.

Rodríguez-González, R., Peteiro, L. M. \& Rodríguez-Wong, M. T. (2007). La educación desde un enfoque histórico social: importancia para el desarrollo humano. Revista Electrónica Psicología Científica. Extraído el 25 de noviembre de 2009 desde http://www.psicologiacientifica.com/ bv/psicologiapdf-255-la-educacion-desde-un-enfoque-historico-social-importancia-para-eldesarrollo-hu.pdf

Sáinz, M., López-Sáez, M. \& Lisbona, A. (2004). Expectativas de rol profesional de mujeres estudiantes de carreras típicamente femeninas o masculinas. Acción Psicológica, vol.3, n.2, 111-123. 